Disponível em

http://www.anpad.org.br/rac

RAC, Curitiba, v. 14, n. 2, art. 7,

pp. 310-332, Mar./Abr. 2010

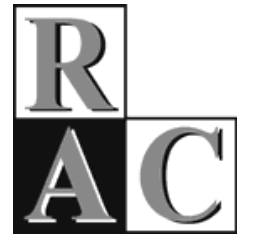

\title{
A Encruzilhada da Aprendizagem Organizacional: uma Visão Multiparadigmática
}

\author{
The Crossroads of Organizational Learning: a Multiparadigmatic View
}

Claudia Simone Antonello* Doutora em Administração pelo PPGA/UFRGS. Professora e pesquisadora do PPGA/UFRGS, Porto Alegre/RS, Brasil.

Arilda Schmidt Godoy Doutora em Educação pela USP. Professora da Universidade Presbiteriana Mackenzie, São Paulo/SP, Brasil.

* Endereço: Claudia Simone Antonello

Rua Washington Luís, 855, sala 315, Centro, Porto Alegre/RS, 90010-460. E-mail: csanotnello@ea.ufrgs.br

Copyright (C) 2010 RAC. Todos os direitos, inclusive de tradução, são reservados. É permitido citar parte de artigos sem autorização prévia desde que seja identificada a fonte. 


\title{
RESUMO
}

A literatura sobre Aprendizagem Organizacional [AO] tem avançado não somente no volume de publicações, mas apontando outras dimensões do tema. Envolve diversos campos teóricos: psicológicos, sociológicos, culturais, históricos e metodológicos, além da gestão propriamente dita. Assim, pergunta-se: Quais questões e características básicas fazem a AO peculiar e a separa de outros conceitos relacionados? Para responder a este questionamento são apresentadas as diversas perspectivas teóricas que abordam AO, algumas questões e reflexões relacionadas às teorias, assim como definições e estudos desenvolvidos no campo. Utilizando-se uma análise de inspiração multiparadigmática, as contribuições identificadas foram agrupadas em cinco campos que apresentam alguns temas e questões recorrentes. Esta análise aponta quatro questões (relacionadas ao nível de análise, resultados da aprendizagem, mudanças e processos de aprendizagem), que o conceito de AO deve superar para tornar-ser significativo. Visando superar estes desafios, sugere-se a adoção de uma perspectiva baseada em práticas.

Palavras-chave: aprendizagem organizacional; aprendizagem baseada em práticas; abordagem multiparadigmática; revisão de literatura; método de pesquisa.

\begin{abstract}
The literature on Organizational Learning [OL] has been moving forward not only in the volume of publications but also by pointing to other dimensions of the theme. The debate involves several theoretical fields psychological, sociological, cultural, historical and methodological - in addition to management itself. A question emerges: which issues and basic characteristics make OL different and separated from the other related concepts? In order to answer this question a number of theoretical perspectives of OL are presented and discussed, along with some inquiries and reflections related to these theories, as well as definitions and studies developed in this field. Using the multiparadigmatic approach, the literature on OL was organized and grouped into five fields. This analysis pointed to four questions (related to the level of learning, learning results, changes and learning processes) that the concept of OL has to overcome to become significant. It is suggested that a possible way to overcome these challenges is to adopt a perspective based on practices.
\end{abstract}

Key words: organizational learning; learning based in practice; multiparadigmatic approach; literature review; research method. 


\section{INTRODUÇÃO}

O conceito de aprendizagem organizacional [AO] difundiu-se tanto na pesquisa em administração, quanto em sua prática. Cada vez mais as publicações orientadas para administradores encorajam que as organizações devam tornar-se organizações que aprendem para sobreviver aos tempos turbulentos do ambiente de negócios (Dragonetti, Antonacopoulou, Broekhuizen, \& Patnaik, 2005). O tema está se tornando tão popular que se ampliou além do discurso da administração para debates mais gerais da política sobre a aprendizagem das nações (Contu, Grey, \& Örtenblad, 2003).

O perigo desta popularidade, que alguns chamariam de superexposição, é a perda de especificidade do próprio conceito. Na realidade, "é provável que o conceito de aprendizagem organizacional permaneça como conceito guarda-chuva para muitos conceitos relacionados” (Argote, 1999, p. 13). Porém o risco é que, ao se tentar abarcar muitos destes conceitos relacionados, a identidade própria da aprendizagem poderá ser perdida. Para se avançar na compreensão da AO, então, tem-se como desafio manter a energia e a vitalidade que se originam das diversas perspectivas com que o assunto tem sido abordado e, ao mesmo tempo, delinear e discutir algumas questões e características básicas que tornam esse conceito peculiar, separando-o de outros a ele relacionados. Este é o objetivo do presente artigo.

O conceito de AO tem uma linhagem muito distinta nos estudos de administração. Na realidade, é quase assustador tentar revisar a literatura, por causa da amplitude de idéias que podem ser consideradas relevantes. A partir de contribuições seminais nos anos 50 e 60 (Cangelosi \& Dill, 1965; March \& Simon, 1993), a pesquisa em AO se ramificou para incluir insights da psicologia, sociologia, ciência da computação e economia.

A literatura sobre AO tem avançado nos últimos tempos não somente no volume de publicações, mas apontando outras dimensões do tema. Ela apresenta um legado de mais de 30 anos, com crescimento exponencial a partir dos anos 90 (Crossan \& Guatto, 1996; Easterby-Smith, 1997). Desde então, diversas revisões de literatura contribuíram para a consolidação e progresso no campo (Dodgson, 1993; Easterby-Smith, 1997; Fiol \& Lyles, 1985; Huber, 1991; Miller, 1996). O estudo de Bontis, Crossan e Hulland (2002) revelou que uma diversidade de perspectivas foi utilizada para olhar o tema AO no período. Essas revisões trabalharam principalmente com a análise teórica e identificaram várias preocupações, particularmente o não acúmulo e a falta de integração dos estudos desenvolvidos, dificultando a geração de norteadores e de uma agenda de pesquisa, além da carência de trabalhos empíricos. Para Lyles e Easterby-Smith (2003) apenas 19\% das publicações nos últimos anos examinaram os métodos utilizados para pesquisar e mensurar $\mathrm{AO}$, o que exige maior reflexão sobre os desenhos de pesquisa relacionados ao tema.

Recentemente, num esforço para responder a estas questões, Bapuji e Crossan (2004) avaliaram a produção de 1990 a 2002 no campo e identificaram, entre outros achados, o aumento em qualidade e quantidade da pesquisa empírica. Ainda, Berthoin Antal, Dierkes, Child e Nonaka (2001) comentam que pouco se sabe sobre a criação de conhecimento em AO nos países da África e da América do Sul, exigindo que se empreenda mais em pesquisas que mapeiem o tema em outros países além dos Estados Unidos e da Europa.

Dada a pluralidade da produção em AO, muitos estudiosos tentaram classificar a literatura no campo em diferentes disciplinas, escolas de pensamento ou perspectivas. Na literatura internacional pode-se mencionar Shrivastava (1983), Wiegand (1996), Tsang (1997), Easterby-Smith (1997), Edmondson e Moingeon (1998), Pawlowsky (2001) e Bell, Whitwell e Lukas (2002); na literatura brasileira foi possível identificar os estudos de Loiola e Bastos (2003) e de Antonello (2005). Ao se examinar tais propostas de sistematização e classificações teóricas evidencia-se que, até mesmo dentro da área, os autores apresentam perspectivas diferentes de AO como base para desenvolver suas idéias.

No Brasil estudos desenvolvidos por Antonello (2002, 2005) e por Loiola e Bastos (2003) tiveram como objetivo identificar e analisar a produção científica brasileira sobre AO no período de 1997 a 2001. Os resultados indicam que a afluência da literatura brasileira sobre AO subsidia o 
esclarecimento de diversos aspectos, mas muitas vezes obscurece informação para pesquisadores e gestores. Os conceitos predominantes recebem uma influência forte da abordagem da administração científica tradicional. É nesse contexto, pela própria abrangência, que o debate apropria diversos campos teóricos: psicológicos, sociológicos, culturais, históricos e metodológicos, além da gestão propriamente.

Estas considerações instigam importante questionamento: Quais questões e características básicas fazem a aprendizagem organizacional peculiar e a separam de outros conceitos relacionados?

Para responder a esta questão o artigo está organizado nas seguintes seções: (2) referencial apresentando as perspectivas teóricas que abordam AO; (3) seção que explicita o enfoque multiparadigmático utilizado na análise da produção apresentada; (4) seção que aborda algumas questões e reflexões relacionadas às teorias, definições e estudos desenvolvidos em AO e, por fim, (5) as considerações finais que apresentam os aspectos essenciais da abordagem baseada em práticas, tendo em vista o seu papel integrador e de reflexividade sobre o campo da AO.

\section{Referencial Teórico: ApresentaÇÃo das Perspectivas}

A escolha das perspectivas aqui apresentadas está sustentada nas coletâneas de textos organizadas por Dierkes, Berthoin Anthal, Child e Nonaka (2001) e Easterby-Smith e Lyles (2003), que reúnem contribuições de reconhecidos pesquisadores. Estas obras resumem e analisam o estado da arte no campo da AO, apresentam diferentes abordagens teóricas, aplicações e práticas, enriquecendo o debate sobre as debilidades e forças dos estudos sobre o tema, além de delinear uma agenda de pesquisa, considerando o contexto global, atores e processos organizacionais. A análise deste material, posteriormente, ocorreu à luz da abordagem multiparadigmática que será comentada em seção específica.

\section{Aprendizagem Organizacional e a Perspectiva Psicológica}

A literatura da administração contemporânea contém basicamente duas visões sobre a relação entre AO e o campo da aprendizagem em psicologia. Na primeira a AO é vista como uma analogia da aprendizagem individual. A partir desta perspectiva, o conhecimento sobre processos de aprendizagem individual pode ser usado para compreender o processo de AO (Walsh \& Ungson, 1991). Na segunda visão, a aprendizagem individual é a base para a AO (Argyris \& Schon, 1978; Cangelosi \& Dill, 1965; Hedberg, 1981). De acordo com esta visão, o incremento do conhecimento organizacional - um indicador de AO - está baseado na aquisição de conhecimentos dos indivíduos na organização.

Como existem inúmeras perspectivas para explicar a aprendizagem individual a partir de uma abordagem psicológica, muitos caminhos e formas de pensamento sobre AO têm sido postulados. Após a análise de 70 artigos, Maier, Prange e Rosentiel (2001) propuseram três visões teóricas para AO: (a) Comportamental/Processamento da Informação; (b) Construção Social; (c) Psicologia Aplicada.

\section{Comportamental}

As teorias comportamentais a respeito da AO focam os antecedentes e as mudanças que ocorrem nas rotinas e nos sistema internos das organizações, quando elas respondem às suas próprias experiências e àquelas de outras organizações. Nelson e Winter (1982) apresentam uma teoria identificada como a mais bem desenvolvida a respeito do tema e que foi por eles nomeada de modelo evolucionário da firma. 
Segundo esta teoria as organizações aprendem, codificando inferências a partir da sua história e das rotinas que guiam o comportamento delas. Estas rotinas independem dos atores individuais que as executam, sendo mantidas mesmo quando há considerável turnover de indivíduos na organização. Dois importantes construtos do modelo são path dependence e trajetória. Path dependence envolve o princípio de que a história da organização é fundamental e que as condições iniciais para criação de uma organização predeterminam o repertório de habilidades individuais e capacidades organizacionais que a impelem a se adaptar às circunstâncias ambientais. Trajetória envolve a noção de que a aprendizagem futura de novas capacidades será construída sobre a aprendizagem acumulada e as capacidades obtidas no passado. Os modelos de AO, segundo esta perspectiva, estão focados nas consequências como fator que mais impacta a aprendizagem. Também se apóiam nos conceitos da psicologia da cognição e na noção psicodinâmica de que toda a aprendizagem é influenciada pelo passado.

Outra vertente das teorias comportamentais refere-se à aprendizagem enquanto sistema de processamento da informação que está inspirada na simulação de computador de padrões complexos das cadeias de evolução de comportamento e suas consequências na performance, descrita por Huber (1991). Este tipo de abordagem implica considerar que a aprendizagem organizacional e o desempenho não podem ser reduzidos à aprendizagem individual ou grupal e ao comportamento, pois vêem a aprendizagem organizacional como fenômeno emergente. Esta vertente apresenta uma visão mais técnica e entende a $\mathrm{AO}$ como um processo efetivo, de interpretação e resposta para informação interna e externa da organização. Esta informação pode ser quantitativa ou qualitativa, mas geralmente é explícita e de domínio público. Uma das exposições mais claras desta visão vem de Huber (1991, p. 89), cuja afirmativa diz: "uma entidade aprende, se através de seu processo de informação a extensão de seu comportamento potencial é mudado ... uma organização aprende, se quaisquer de suas unidades adquire conhecimento que é reconhecido como potencialmente útil para a organização”.

\section{Construção Social}

Esta perspectiva focaliza a AO, considerando os processos cognitivos socialmente mediados pela interpretação e o sensemaking, enfatizando o contexto social. A aprendizagem é socialmente construída e fundamentada em situações concretas, nas quais as pessoas participam e interagem umas com as outras. Esta perspectiva assume que a aprendizagem está incrustada nas relações e interações das pessoas (Lave \& Wenger, 1991; Orr, 1990). Em síntese a perspectiva da construção social delineia mais explicitamente seus conceitos e tem suas raízes na perspectiva da psicologia sociocultural. Nesta abordagem os aspectos situacionais da aprendizagem têm papel central, enquanto os princípios psicológicos da cognição social e individual são considerados processos secundários.

Dois modelos de AO são desenvolvidos a partir da idéia de produção de sentido: comunidades de prática (Cop’s de Brown \& Duguid, 1991; Lave \& Wenger, 1991) e o do SECI (criação do conhecimento de Nonaka \& Takeuchi, 1997). Os estudos sobre Cop’s de Brown e Duguid (1991) revelam estar fortemente fundamentados na psicologia sociocultural e nas teorias cognitivas sociais de aprendizagem. De forma contrastante, os estudos sobre SECI se inspiram nas teorias da construção social, mas parecem não ter diretamente raízes na psicologia sociocultural e da cognição social de aprendizagem.

\section{Psicologia Aplicada}

Aqui adota-se a noção de que a AO está fundamentada na experiência direta e também requer ativa intervenção dos facilitadores em treinamento e dos consultores para incrementar as práticas dos indivíduos e das organizações. Muito da teoria da aprendizagem na ação parte do modelo de aprendizagem experiencial de Kolb (1984), inspirado em Piaget (1978), Lewin (1947) e Dewey (1938). Similarmente, diversos consultores e teóricos da aprendizagem na ação (entre eles Raelin, 2001) citam Mezirow (1991) e suas idéias sobre reflexão, baseadas na psicologia do desenvolvimento cognitivo. 
Nesta perspectiva há uma vertente designada action learning (Revans, 1982), que propõe que a aprendizagem resulta da interação do questionamento programado com o espontâneo, considerando a experiência vivida. A intervenção em action learning enfatiza a reflexão sobre o comportamento na solução de problemas e constrói suposições sobre o que interfere no trabalho efetivo e na aprendizagem individual. Já a Ciência Ação é um tipo de técnica investigativa desenvolvida a partir do trabalho de Kurt Lewin (1947) e popularizada por Argyris e Schön (1978). Está baseada em diferentes perspectivas psicológicas e parte do princípio de que as pessoas podem melhorar sua eficiência interpessoal e organizacional a partir da análise das crenças latentes que bloqueiam suas ações.

\section{Aprendizagem Organizacional e a Perspectiva Sociológica}

De acordo com Gherardi e Nicolini (2001, p. 47), os "sociólogos não abordam a aprendizagem como algo que acontece na mente, mas como algo produzido e reproduzido nas relações sociais dos indivíduos, quando eles participam de uma sociedade”. Este conceito sugere que a aprendizagem está integrada no cotidiano da vida dos indivíduos. Sugere também que grande parte da aprendizagem seja oriunda da fonte informal das relações sociais, o que introduz, posteriormente, o conceito de prática como fator proeminente na visão sociológica. Gherardi e Nicolini (2001, p. 49) comentam que, "prática é um sistema de atividades nas quais saber não está separado do fazer e das situações e poderia ser chamado de conhecimento coproduzido por meio da atividade”. Pressupõe que toda atividade na vida dos indivíduos é uma oportunidade para aprendizagem e que a aprendizagem em situações sociais casuais é tão importante quanto experiências de aprendizagem formais.

Collins (1994) sugeriu quatro tradições sociológicas e Gherardi e Nicolini (2001) acrescentaram o pós-modernismo que, embora não possa ser considerado tradição, pode estimular a reflexão sobre o conhecimento enquanto prática discursiva. A partir destas tradições são estabelecidas narrativas de AO, as quais são apresentadas na Tabela 1.

Tabela 1

As Tradições Sociológicas e as Narrativas de Aprendizagem Organizacional

\begin{tabular}{cl}
\hline Tradição Sociológica & \multicolumn{1}{c}{ Narrativa de Aprendizagem organizacional } \\
\hline Tradição do Conflito & - AO como ideologia de um grupo de poder. \\
(Marx, Engels, Weber) & - AO como política de mobilização de recursos de poder e conflito \\
& - AO como tentativa de gerenciar a tensão entre a racionalidade substantiva e a \\
& formal. \\
Racional/Utilitária & - AO como resolução de problemas, quando o desempenho da organização não \\
(Humans, Blau, Cook, & conhece os níveis de aspiração. \\
Simon) & AO como a ativação da troca no trabalho em rede. \\
& AO como aprendizagem ecológica, localizada e distribuída pelos multiatores \\
& dentro das rotinas, ao invés de dentro da mente dos indivíduos. \\
Durkheiniana & AO como uma variável dependente de outras variáveis (estratégia, estrutura ou \\
(Durkheim) & cultura) define as condições que facilitam ou dificultam a aprendizagem \\
& organizacional. A aprendizagem é compreendida como uma das funções do \\
& sistema organizacional que engendram mudança sobre algumas ocasiões e \\
& conservam sobre outras. \\
& AO como socialização de códigos culturais específicos. Conceitua \\
& aprendizagem organizacional como socialização, que sintetiza conteúdos de \\
diferentes ordens da sociedade, porque as ações seletivas em sua chance de \\
vida criam um senso de determinar inevitavelmente ordem social e restringir o \\
resultado de mudança que é permitida.
\end{tabular}

Continua 


\section{Tabela 1 (Continuação)}

\begin{tabular}{cc}
\hline Tradição Sociológica & Narrativa de Aprendizagem organizacional \\
\hline Microinteracionista & - AO como transmissão de conhecimento dentro das comunidades ocupacionais \\
(Peirce, Mead, Hurssel, & - AO como rótulo que produz uma realidade socialmente construída e é \\
Schutz e Garfinkel) & produzida por esta realidade. \\
Pós-Moderna & - AO como prática discursiva
\end{tabular}

Nota. Fonte: adaptado de Gherardi, S., \& Nicolini, D. (2001). The sociological foundations of organizational learning (p. 46). In M. Dierkes, A. Berthoin Antal, J. Child, \& I. Nonaka (Orgs.), The handbook of organizational learning and knowledge (pp.35-60). Oxford: Oxford University Press.

A sociologia descreve a aprendizagem social como processo que envolve relações sociais, processo formado por instituições sociais; e a aprendizagem em si mesma: objeto cultural criado por práticas hábeis de trabalho cultural. Quando se utiliza a ontologia construcionista, a AO é percebida como conversação aberta; dois importantes conceitos podem contribuir para isto: a participação e a reflexividade.

O conceito de participação lança luzes no fato de que a aprendizagem não ocorre somente na mente dos indivíduos, mas origina-se e sustenta-se na participação deles em atividades sociais. A lógica da prática não traça distinção entre conteúdo e objeto; ao invés disto, envolve o saber como ser competente num ambiente. Este conceito conduz ao estudo do conhecimento pré-reflexivo, o conhecimento tácito, a compreensão estética, o conhecimento em ação: enfim o que alguns autores denominam learning-organizing.

A reflexividade, em contraste, relata o momento particular da separação entre o conteúdo do conhecimento e o seu objeto do conhecimento. Isto instiga a organização, envolvida no processo social, a extrair o conhecimento teórico oriundo do conhecimento prático e, então, transformá-lo em conhecimento normativo, produzindo as condições necessárias para a operacionalização deste conhecimento (Lave \& Wenger, 1991; Tenkasi \& Boland, 1996).

A principal característica da reflexividade é que a reflexão produz o setting que contém estes objetos, bem como os resultados esperados e os não esperados. O conceito de reflexividade está proximamente vinculado à participação; a reflexividade ocorre quando o fluxo da experiência é interrompido e os conteúdos refletem sobre o conhecimento. Refere-se às maneiras de ver as ações anteriores e refletir sobre a existência de outras formas de vê-las. A reflexividade dá origem à institucionalização do conhecimento.

\section{Aprendizagem Organizacional e a Perspectiva Antropológica}

Uma maneira de construir a ponte entre a antropologia e a teoria das organizações foi a de conceber a organização como processos, ao invés de como estruturas. Além disso, os próprios antropólogos providenciaram argumentos para tratar esta questão: "Culturas são organizações para fazer algo, para perpetuar a vida humana e a si mesmas” (Czarniawska, 2001, p. 24).

A antropologia começou a utilizar o termo AO como referência à aprendizagem que o indivíduo realiza em contexto de estruturas sociais. O conceito entrecruza diferentes disciplinas e está focalizado na maneira como as pessoas aprendem em estruturas sociais. A natureza e o processo da aprendizagem podem variar em culturas e situações diferentes.

Segundo Easterby-Smith (1997), estes aspectos associados à globalização crescente dos negócios e da administração trouxeram uma necessidade urgente de desenvolver visões mais localizadas da AO. As contribuições da perspectiva antropológica seriam: (a) atribuir atenção para a importância de valores e convicções; (b) apresentar como a cultura pode afetar o processo e a natureza da AO; e (c) examinar a dificuldade de transferir idéias de uma cultura para outra. O foco está no coletivo, nas ações (incluindo 
interações), nos objetos que são foco destas ações, na linguagem utilizada nestas ações, juntamente com os significados de terreno específico destes vários artefatos para os atores na situação.

\section{Aprendizagem Organizacional e a Perspectiva da Ciência Política}

A ciência política tem como conceitos centrais a natureza, a posse e o exercício do poder. Estes conceitos tendem a ser obscurecidos e a transcender muito do que ocorre na esfera pública/privada. Este fato é criticamente importante, considerando-se que seu tratamento é extenso. Neste campo a AO tem atraído pouca atenção dos cientistas políticos.

No entanto a pesquisa da aprendizagem na área do setor público se constitui numa contribuição do campo de ciência política para a AO. A mudança de governos, por exemplo, pode exercer pressão para mudanças de propriedade e de controle, especialmente por meio da privatização, que tende a desencadear novas políticas organizacionais com implicações para as formas e a identidade da organização (Rodrigues, Child, \& Luz, 2003). Desse modo, a ideologia política tem impacto nas organizações por meio de decisões sobre formas de organização - hierarquia e estrutura - e estratégia. A ideologia, porém, não é simplesmente força autônoma, exógena.

Aprendizagem é percebida como processo político no qual os atores investem muitos esforços para influenciar uns aos outros, interpretar a experiência e tentar construir coalizões internas. O framework conceitual pelo qual a experiência é interpretada, a comunicação estruturada e a informação difundida, torna-se muito importante. Aprendizagem é vista como luta interna para o desenvolvimento de novos modelos mentais compartilhados e uma ênfase é atribuída ao conflito intraorganizacional, isto é, o conflito entre subgrupos ou partes com interesses políticos diferentes.

Embora alguma atenção tenha sido dada à associação entre a $\mathrm{AO}$ e a mudança organizacional, somente alguns estudos aprofundaram a questão (Lapalombara, 2001), no sentido de tentar explicar como as organizações absorvem as visões e opiniões de que estão imbuídas ideológica e institucionalmente e as transformam em práticas e rotinas.

Os estudos sobre o controle administrativo e a subjetividade no local de trabalho trouxeram os temas de ideologia e aprendizagem à atenção (Alvesson \& Willmott, 2002); mas com pouca referência à influência potencial do ambiente institucional. Alguns estudos foram desenvolvidos, demonstrando como a aprendizagem afeta os significados intersubjetivos e a identidade (Corley \& Gioia, 2003; Gherardi \& Nicolini, 2001); entretanto essas contribuições não investigaram como a ideologia pode associar as identidades das organizações e dos indivíduos às relações de poder por meio dos processos de aprendizagem.

\section{Aprendizagem Organizacional e a Perspectiva Histórica}

Conforme Weber e Berthoin Antal (2001, p. 358) a "história tem um efeito de identificação para organizações”. A forma como uma organização empregou a aprendizagem no passado pode ser utilizada em oportunidades futuras. A história ou a identidade de uma organização é construída em parte sobre a aprendizagem coletiva dos indivíduos e grupos dentro da organização, no transcorrer do tempo. Estes autores dizem que "a influência da história na organização pode ser positiva como também disfuncional” (p. 358). Organizações podem usar da sua história para ampliar o potencial de seu conhecimento coletivo armazenado. Porém elas têm de se precaver da obsolescência que pode ocorrer com a aderência rígida às práticas e procedimentos passados, sem a consideração de nova aprendizagem e oportunidades.

As narrativas podem oferecer um senso do processo desregrado de aprendizagem organizacional, superando instantaneamente a abordagem denominada de antes-depois e de entrada-saída. A aprendizagem organizacional é um meio de reimaginar a história e a natureza da imagem da organização. As histórias podem revelar alternativas do passado ou do presente não consideradas pela organização. Por exemplo, buscar identificar as razões originais e as decisões para entrar num 
determinado mercado ou desenvolver produtos pode fornecer descobertas sobre trajetórias alternativas para as organizações (Fear, 2001).

Há uma corrente de historiadores que considera a reconstrução do passado e a redescoberta dos significados e suposições que guiam os comportamentos e ações das pessoas ao longo do tempo. Os significados e a linguagem contemporâneos auxiliam a construir pensamentos e decisões prévias no tempo.

Esta perspectiva pode criar um distanciamento das presentes práticas, ensejando a reflexividade e a aprendizagem. Este distanciamento pode ter mais valor do que as lições de aprendizagem obtidas a partir das histórias, lições que podem parecer mais conclusivas, mas podem impedir o processo de reflexão no momento atual. Ela dá ensejo a uma abordagem vicária ou por contraste, desenvolvendo um senso de como categorias e significados, muitas vezes, vão alterando-se no transcorrer do tempo. Ilumina o presente organizacional, examinando a trajetória do uso da linguagem: metáforas, símbolos e cultura presente. Assim, sugere dar-se menos importância à descrição e análise do processo em si mesmo e voltar-se para a detecção de quando os problemas se tornam perceptíveis, como as organizações reagem a mudanças do ambiente, quando tentam mudar e como superam ou não estas mudanças.

\section{Aprendizagem Organizacional e a Perspectiva Econômica}

Nesta perspectiva a aprendizagem é definida como a aquisição e uso do conhecimento existente e/ou a criação de novo conhecimento com o propósito de melhorar o desempenho econômico (Boerner, Macher, \& Teece, 2001). Apesar da centralidade da aprendizagem em questões econômicas fundamentais, seu tratamento na literatura da economia ortodoxa é escasso e, frequentemente, caricaturado.

De certa forma, este descaso com aprendizagem organizacional na economia não surpreende. A negligência em relação à aprendizagem em economia sustenta-se, em parte, pelo fato dos teóricos construírem suas suposições a partir dos processos de comportamento e tomada de decisão dos agentes econômicos. Em ambiente baseado nestas suposições, os agentes são perfeitamente racionais e capazes de responder ótima e instantaneamente a condições variáveis. A natureza estilizada de tal ambiente é resumida por Nelson e Winter (1982).

Admite-se que é difícil definir e diferenciar uma abordagem econômica da de aprendizagem. Boerner et al. (2001) propõem sete abordagens que contemplam os modelos mais proeminentes de aprendizagem identificados na literatura ortodoxa em economia. Estas abordagens são empregadas para modificar as suposições tradicionais e os temas de estudos relacionados à aprendizagem (vide Tabela 2).

Tabela 2

Modificações na Ortodoxia para Acomodar a Aprendizagem

\begin{tabular}{|c|c|c|c|}
\hline & $\begin{array}{c}\text { Suposições } \\
\text { Comportamentais }\end{array}$ & $\begin{array}{l}\text { Ambiente } \\
\text { Institucional }\end{array}$ & Conceito de Equilíbrio \\
\hline \multirow[t]{2}{*}{ Ortodoxa } & Hiperracionalidade & Ajustamento sem atrito & Ajustamento instantâneo \\
\hline & Certeza & & \\
\hline \multirow{3}{*}{$\begin{array}{l}\text { Modificações } \\
\text { sugeridas } \\
\text { para } \\
\text { ortodoxia }\end{array}$} & (1) Racionalidade Limitada & (3) Aprendizagem Social & (5) Abordagem da Teoria dos jogos \\
\hline & (2) Incerteza & (4) Path Dependency & (6) Ajustamento Bayesiano \\
\hline & & & $\begin{array}{l}\text { (7) Learning-by-doing e Curvas de } \\
\text { Aprendizagem }\end{array}$ \\
\hline
\end{tabular}

Nota. Fonte: Boerner, C., Macher, J., \& Teece, D. (2001). A review and assessment of organizational learning in economic theories (p. 92). In M. Dierkes, A. Berthoin Antal, J. Child, \& I. Nonaka (Orgs.), The handbook of organizational learning and knowledge (pp. 89-117). Oxford: Oxford University Press. 
O tratamento de AO tem sido explorado em dois campos relativamente influentes da economia: a economia de inovação e a teoria da firma. Estes dois fluxos de literatura proveem novos e importantes insights acerca dos processos de aprendizagem individuais e de como a aprendizagem individual é transformada em AO. Eles também ampliam a ortodoxia não só examinando como empresas adquirem e utilizam conhecimento existente, mas também como elas criam novo conhecimento. Esta extensão é essencial para o progresso de um framework mais inclusivo e mais dinâmico para entender a $\mathrm{AO}$ que existe hoje.

Um tema central da literatura de inovação traz que os meios pelos quais as empresas desenvolvem um novo produto e tecnologias de processo não são fortuitos; muito da inovação tecnológica é estruturada e ordenada e acontece dentro de estruturas bem definidas (Dosi \& Orsenigo, 1988; Pavitt, 1987).

Em esforço para melhorar a compreensão destas estruturas, pesquisadores da inovação colocaram ênfase considerável nos processos de AO. Os insights obtidos por estes estudiosos ampliaram o tratamento da aprendizagem na literatura da economia. Visto sob esta ótica, toda inovação tecnológica requer $\mathrm{AO}$.

Embora qualquer tentativa para resumir a contribuição da literatura de inovação para a AO em poucas páginas seja inadequada, as principais contribuições são resumidamente apresentadas na Tabela 3.

Tabela 3

\section{Categorias de Aprendizagem na Literatura de Inovação}

\begin{tabular}{|c|c|c|}
\hline Tipo de Aprendizagem & Locus & Foco da Inovação \\
\hline Learning-by-doing & Interno à empresa & Atividades de produção \\
\hline Learning-by-searching & Principalmente interno à empresa & $\begin{array}{l}\text { Foco principalmente comercial - P\&D - } \\
\text { relacionado }\end{array}$ \\
\hline Aprendizagem Científica & Interno e externo à empresa & Conhecimento tecnológico \\
\hline Learning-by-using & Interno à empresa & Uso de produtos e inputs \\
\hline Spillover learning & Externo à empresa & $\begin{array}{l}\text { Absorvendo conhecimento externo e } \\
\text { imitando as práticas de concorrentes }\end{array}$ \\
\hline
\end{tabular}

Nota. Fonte: adaptado de Boerner, C., Macher, J., \& Teece, D. (2001). A review and assessment of organizational learning in economic theories (p. 104). In M. Dierkes, A. Berthoin Antal, J. Child, \& I. Nonaka (Orgs.), The handbook of organizational learning and knowledge (pp. 89-117). Oxford: Oxford University Press.

Os pesquisadores que estudam a economia da inovação, a exemplo de Nelson e Winter (1982) e Dosi e Orsenigo (1988), realçaram os vários tipos de aprendizagem que as organizações empregam e observaram que as organizações tiram proveito de várias fontes internas e externas de aprendizagem. Esta literatura também aponta a natureza incremental e cumulativa da aprendizagem e inovação.

Os economistas que trabalham na perspectiva da teoria da firma - Teorias Baseadas em Recursos e Teorias Evolucionárias da Firma, inspirados nos trabalhos de Coase (1937), Hayek (1945), Penrose (1959), Prahalad e Hamel (1990) - utilizam-se dos insights sobre a natureza e as fontes de aprendizagem para explorar como processos internos da firma afetam a habilidade das organizações econômicas desenvolverem e explorarem novas e existentes formas de conhecimento: Capacidades dinâmicas e a Criação do Conhecimento na Organização. O que emerge da economia, entretanto, não é retrato completo de $\mathrm{AO}$, mas fotografias instantâneas úteis de vários aspectos críticos dos processos de aprendizagem. 


\section{Aprendizagem Organizacional e a Perspectiva da Ciência da Administração}

A partir de revisão da literatura sobre as perspectivas e abordagens teóricas para a questão da mudança organizacional e a AO, Pawlowsky (2001) propõe uma sistematização de abordagens e um modelo que procura auxiliar a ciência da administração e o gerenciamento da aprendizagem nas organizações. As diferentes abordagens para AO estão arraigadas numa variedade de estruturas teóricas e dificilmente haverá plataforma teórica que possa servir como base comum.

Pawlowsky (2001) classifica AO em cinco perspectivas teóricas. No entanto, seguindo a linha de raciocínio deste autor e, a partir da revisão de literatura sobre AO, identificou-se que está emergindo uma sexta perspectiva que poderia ser denominada de Perspectiva da Estratégia/Gerencial. Estas seis perspectivas serão apresentadas a seguir.

\section{(a) Perspectiva Cognitiva e do Conhecimento}

Os pesquisadores que adotam esta perspectiva tendem a pensar em AO como tendo uma base cognitiva na qual o conhecimento é acumulado de modo consciente. Durante o processo de AO a informação tem papel essencial. Para Argyris e Schön $(1978$, p. 10) "toda ação deliberada teve uma base cognitiva, que reflete normas, estratégias e suposições ou modelos do mundo ... e aprendizagem individual pela aquisição do conhecimento cria uma fundação para aprendizagem organizacional”.

Na mesma linha, Slater e Narver (1995) definem aprendizagem como o desenvolvimento de novos conhecimentos e insights que têm influência no comportamento. Assim, o processo de aprendizagem inclui a aquisição e disseminação da informação e interpretação compartilhada. Conforme Pawlowsky (2001), outros pesquisadores que adotam esta abordagem são Huber (1991), Fiol e Lyles (1985) e Garvin (1993).

A noção essencial da perspectiva cognitiva é que os membros das organizações não são somente uma caixa de armazenamento de experiências racionais passadas, mas intérpretes da realidade de acordo com as especificidades do seu sistema cognitivo. A perspectiva cognitiva e do conhecimento da AO também inclui uma variedade de agrupamentos, podendo-se distinguir duas abordagens que têm recebido considerável interesse.

A primeira engloba as abordagens estruturais também denominadas como Representacionismo e tem como foco as habilidades de processamento da informação que dependem de características estruturais do sistema cognitivo (Huber, 1991).

A segunda, de caráter epistemológico, considera o processo de interpretação e a construção cognitiva de realidade como tema central de importância para a aprendizagem (Daft \& Weick, 1984; Krogh, Roos, \& Slocum, 1996).

\section{(b) Perspectiva da Tomada de Decisão Organizacional e da Adaptação}

Esta perspectiva está bem representada pelo trabalho de Cyert e March (1992), que teve como foco o nível organizacional da aprendizagem por adaptação. Nele os autores entendem que

organizações exibem comportamento adaptável com o passar do tempo. Da mesma maneira que adaptações em nível individual dependem de fenômenos da fisiologia humana, adaptação organizacional usa os indivíduos da organização como instrumentos. Porém, nós acreditamos que é possível lidar com adaptação em nível agregado da organização (Cyert \& March, 1992, p. 13).

Portanto as idéias desta perspectiva estão alinhadas com as teorias comportamentais a respeito de AO já expostas no item Aprendizagem Organizacional e a Perspectiva Psicológica. Porém ocorre um avanço nesta abordagem, à medida que Levitt e March (1988) explicitam o significado do termo 
rotinas, incorporando ao mesmo as formas, regras, procedimentos, convenções, estratégias, tecnologia em torno das quais as organizações são construídas e por meio das quais elas operam.

\section{(c) Perspectiva da Teoria de Sistemas}

A Perspectiva da Teoria de Sistemas para AO define a organização como sistemas de atividades conscientemente coordenadas. AO é concebida como um incremento na solução de problemas potenciais de sistemas sociais, incremento derivado da aprendizagem institucional.

Senge (1990) propõe a abordagem da dinâmica de sistemas para a AO. A suposição básica é de que, uma vez que se reduza a complexidade de um sistema de rede, analisando as características de todos os fatores e as suas relações dinâmicas com o passar do tempo, este conhecimento pode ser usado para compreender o funcionamento das redes de sistemas complexos e, assim, pode-se intervir adequadamente. Todos os outputs de um sistema são vistos como inputs para outros sistemas, enquanto a aprendizagem significa compreender as relações complexas dos sistemas sociais e a sua dinâmica.

Esta abordagem, baseada em sistemas dinâmicos, foi desenvolvida, entre outros, por Forrester (1975). O conceito foi adotado por Senge (1990) e pela escola de Gallen em desenvolvimento organizacional (Ulrich \& Probst, 1990).

\section{(d) Perspectiva Cultural}

De forma geral, pode-se identificar no mínimo duas grandes vertentes para esta perspectiva. A primeira entende a AO como mudança nas rotinas defensivas no interior das organizações. Argyris (1990) diz que o mecanismo de defesa organizacional relativo aos processos de aprendizagem é fundamentado em nível cultural e emocional da organização. Ele distinguiu os dois tipos contrastantes de culturas nas organizações, que são Modelo I e Modelo II: Teoria em Uso e Teoria Esposada. Num nível conceitual mais simples "nós podemos dizer que cultura é a aprendizagem comum compartilhada e produzida” (Schein, 1991, p. 247).

A outra vertente, tendo como seus principais representantes Cook e Yanow (1993), propõe uma visão distinta da abordagem cultural para a $\mathrm{AO}$, entendendo-a como complemento, não substituto para a perspectiva cognitiva. Para eles a perspectiva cognitiva só focaliza no nível individual, enquanto, pela perspectiva cultural, se pode capturar a aprendizagem em nível de aprendizagem coletivo. Assim, a AO é vista como processo "quando um grupo adquire a experiência que permite levar a cabo suas atividades coletivas” (p. 13).

\section{(e) Perspectiva da Aprendizagem na Ação}

Segundo a Perspectiva da Aprendizagem na Ação (inspirada nas bases teóricas da aprendizagem experiencial (Argyris \& Schön, 1978; Kolb, 1984) acredita-se que a intervenção orientada para o comportamento é o ponto de partida para os processos de aprendizagem. A AO é definida como a capacidade de uma organização para manter ou melhorar seu desempenho baseado na experiência. Aprendizagem ocorre a partir da experiência, e a experiência dos aprendizes é refletida enquanto ocorre a ação.

O framework aprender fazendo é de fato o argumento dos pesquisadores nesta perspectiva, sendo que o conhecimento tácito é um elemento importante durante esse processo. A abordagem da aprendizagem na ação é heterogênea em suas teorias e seus modelos. Enquanto alguns estão baseados no ciclo de aprendizagem experiencial de Kolb, outros fundamentam-se na abordagem das práticas de AO de Revans (1980) e Pedler, Burgoyne e Boydell (1991). 


\section{(f) Perspectiva da Estratégia/ Gerencial}

Finalmente, há uma tendência emergente na literatura de AO denominada Perspectiva da Estratégia/Gerencial. A aprendizagem nesta visão é uma fonte de heterogeneidade entre organizações, criando a base para uma possível vantagem competitiva. A aprendizagem não somente significa que uma organização aprende, mas deve aprender mais rapidamente que outras. Os autores que seguem esta abordagem acreditam que os gerentes têm papel ativo e importante no processo de AO, criando ambiente de aprendizagem dentro da organização, identificando problemas e tomando decisões estratégicas.

\section{A ABORDAGEM MuLTIPARADIGMÁTICA}

Para Lewis e Grimes (2005), é possível identificar na literatura sobre a investigação multiparadigmática três abordagens denominadas: revisões multiparadigmáticas, pesquisa multiparadigmática e construção multiparadigmática de teorias, as quais podem ser entendidas como complementares e que envolvem estágios cada vez mais avançados de reflexão teórica.

De acordo com Gioia e Prite (1990, p. 599) a adoção de uma perspectiva multiparadigmática trará como benefício o desenvolvimento de "uma forma de ver possíveis ligações entre teorias e de justapor ou mesclar perspectivas teóricas alternativas dentro de uma visão teórica multifacetada do fenômeno organizacional”. Para Lewis e Grimes (2005, p. 84) análises deste tipo "dão suporte e elaboram visões díspares, aprofundando a compreensão dos teóricos sobre o fenômeno e os paradigmas empregados." Sugerem que se transcendam os dualismos paradigmáticos e se assuma um pensamento paradoxal, a partir do qual se examinem, simultaneamente, visões conflitantes.

A análise aqui desenvolvida alinha-se à abordagem que Lewis e Grimes (2005) denominam de revisão multiparadigmática e envolve a identificação de agrupamentos e de ligações entre teorias existentes. Busca explicitar premissas divergentes e distinções paradigmáticas, promovendo a compreensão, a utilização e a crítica de perspectivas alternativas. Neste tipo de revisão procura-se trabalhar a partir da descoberta de zonas de transição "que integram interpretações paradigmáticas e enfatizam similaridades entre os paradigmas, podendo privilegiar algum lado numa situação dualista" (pp. 74-75), realçando a importância da comunicação entre paradigmas.

Já existe um número significativo de textos que apontam o domínio dos paradigmas positivista e pós-positivista nas pesquisas que tratam de assuntos relativos à administração e organizações e o desafio das alternativas representadas pelas perspectivas interpretativa e pós-moderna. Embora seja comum associar o uso da expressão pesquisa quantitativa aos paradigmas positivista e póspositivista, e pesquisa qualitativa aos paradigmas interpretativo e pós-moderno, esta relação, de acordo com Bryman (2004), deve ser entendida mais como tendência e não como determinação.

Gephart (1999) também chama atenção para essa questão e ressalta que muitos autores usam aspectos de mais de um paradigma em seus estudos empíricos. Afirma que distinções entre paradigmas não deveriam estar centradas na defesa de um deles em relação a outros, mas no esclarecimento das diferentes formas de pensar e pesquisar, as quais podem ser combinadas em novos e interessantes caminhos, compondo delineamentos consistentes e coerentes.

No estudo de Schultz e Hatch (1996), que examinou a temática cultura organizacional a partir dos paradigmas funcionalista e interpretativo, os autores comentam que este tipo de pesquisa multiparadigmática deveria ser adotada no exame de outros campos, tais como a teoria institucional, identidade organizacional, aprendizagem e cognição. Nos campos da Aprendizagem Organizacional e Gestão do Conhecimento, Vera e Crossan (2003) apontam que futuros trabalhos teóricos deveriam examinar as múltiplas visões que têm sido criadas por diferentes paradigmas, procurando alcançar uma 
abordagem multiparadigmática que faça esforço de estabelecer relações entre elas, conforme recomendam Gioia e Pitre (1990).

Acredita-se, então, que esta abordagem multiparadigmática é apropriada para um campo de conhecimento como o da Aprendizagem Organizacional, no final das contas, multidisciplinar.

Desta forma, com o intuito de tentar responder à indagação central do estudo - quais questões e características básicas fazem a aprendizagem organizacional peculiar e a separam de outros conceitos relacionados? - realizou-se uma análise multiparadigmática das publicações que abordam as perspectivas da Aprendizagem Organizacional presentes nas obras de organizadas por Dierkes et al. (2001) e Easterby-Smith e Lyles (2003), que será apresentada na seção a seguir.

\section{A literatura da Aprendizagem Organizacional em ANálise: Algumas POSSIBILIDADES E REFLEXÕES}

Nesta seção apresenta-se o que foi identificado como contribuições significativas das perspectivas da AO na literatura pesquisada sobre o assunto. Pela análise e crítica dos cinco grupos que inicialmente foram identificados de acordo com suas contribuições, apontam-se quatro questões relevantes que deveriam ser consideradas nos estudos desenvolvidos no campo da Aprendizagem Organizacional. No final discute-se a abordagem da aprendizagem baseada em práticas como uma possibilidade de resposta as estas quatro questões.

\section{As Diferentes Perspectivas da Aprendizagem e suas Contribuições}

Analisando as perspectivas, pode-se dizer que, em sua história, a AO foi caracterizada como incremento/melhoria de desempenho no transcorrer do tempo (Adler \& Clark, 1991; Argote, 1999), fenômeno comportamental (Cyert \& March, 1992; Nelson \& Winter, 1982); fenômeno cognitivo (Lant, 2002; Winter, 2000), fenômeno sociocultural (Cook \& Yanow, 1993; Weick \& Westley, 1996), fenômeno com dimensões reflexivas e emocionais (Antonacopoulou \& Gabriel, 2001; Schön, 1983); fenômeno experiencial (ciclos de aprendizagem e aprendizagem na ação, Argyris \& Schön, 1978; Kolb, 1984) e, recentemente, pela visão de aprendizagem baseada em práticas (Nicolini, Gherardi, \& Yanow, 2003b).

A partir das perspectivas, apresentadas nas seções anteriores, também se poderia dizer que é possível agrupar tais contribuições em cinco grupos: (a) Curvas de aprendizagem, (b) Aprendizagem comportamental, (c) Aprendizagem cognitiva, (d) Aprendizagem pela ação, (e) Aprendizagem social.

Embora tais grupos se orientem por diferentes arcabouços teóricos, é possível identificar pontos de interseção e apontar alguns temas e questões que parecem ser mais bem especificadas e examinadas a partir de uma perspectiva multiparadigmática.

Seguindo esta orientação, ao contrário de outras revisões sobre AO, não se está aqui procurando elaborar ou criar um modelo integrado de aprendizagem. Ao invés de tentar ajustar todas as partes num mesmo conjunto, procurou-se identificar exigências mínimas do conceito de aprendizagem organizacional, baseando-se na literatura e sua crítica existente. Também não se procurou organizar a revisão da literatura por meio de disciplinas (como Easterby-Smith, 1997), preferindo-se agrupar contribuições em abordagens gerais.

Deste modo, identificaram-se macro-grupos, em que visões múltiplas coexistem: por exemplo, a escola comportamental de aprendizagem é desenhada a partir da economia evolutiva (Nelson \& Winter, 1982), da cibernética e da teoria de sistemas (Cyert \& March, 1992), e da sociologia (Feldman \& Pentland, 2003). 
Os cinco grupos em AO identificados na presente revisão não podem ser vistos, como é freqüentemente realizado, como cortes claros da forma aqui exposta. Algumas contribuições ultrapassam os limites e, recentemente, houve um esforço particular para integrar a visão comportamental com a visão cognitiva de aprendizagem (Gavetti \& Levinthal, 2000; Winter, 2000). Outras tentativas de trabalho, mais ambiciosas, buscam integrar todas as perspectivas, pelo link (cognitivo) dos processos de intuição em nível individual com os processos de interpretação e integração em nível de grupo e, finalmente, a institucionalização em nível organizacional; conectam tudo, então, à ação na forma de renovação estratégica (Crossan \& Berdrow, 2003; Crossan, Lane, \& White, 1999; Lawrence, Mauws, Dyck, \& Kleysen, 2005; Pawlowsky, 2001).

A maior parte dos autores, porém, permanece principalmente dentro de uma categoria. Contudo o que se percebe, em alguns casos, é que os estudos e teorias propostas para AO pecam por não levarem em conta as lições oriundas da experiência e dos diversos estudos já desenvolvidos na área de conhecimento em aprendizagem.

\section{A Discussão acerca das Principais Questões e Características Identificadas na Literatura de Aprendizagem Organizacional}

Em função da evolução das teorias e adotando-se um olhar capaz de acomodar diversas representações da temática, acredita-se que seja possível identificar quatro características da AO, que precisam ser mais discutidas, incorporadas e/ou resgatadas para uma compreensão do fenômeno e para o delineamento de um conceito de AO que fundamente seu estudo empírico. Torna-se, assim, importante que se reflita sobre a pesquisa existente em AO, lançando luzes sobre os resultados estabelecidos e as questões que permanecem com necessidade de maior investigação. Pela análise e crítica dos cinco grupos anteriormente identificados, apontam-se quatro questões principais - e relacionadas - para discussão.

1) Nível da Aprendizagem: dever-se-ia levar em conta o fato de que AO recorre, independentemente do nível em questão, ao indivíduo e suas interações: grupal, intergrupal, organizacional e interorganizacional, portanto em nível interpessoal.

2) Neutralidade da meta: a aprendizagem pode ser vista como algo bom ou ruim.

3) Noção de mudança: nem toda a aprendizagem se manifesta como mudança no comportamento.

4) Natureza Processual da aprendizagem: aprendizagem é processo e como tal deveria ser estudada.

A primeira questão trata da discussão sobre os níveis de aprendizagem e levanta-se a possibilidade de considerar a aprendizagem explicitamente como fenômeno interpessoal. Sugere-se que isto pode ser realizado a partir da análise explícita do nível grupal ou organizacional, ou considerando as ligações entre os vários níveis de análise. O que precisa estar presente, entretanto, é uma razão para a separação da aprendizagem individual e um mecanismo que explicaria como grupos e organizações aprendem.

De acordo com Dragonetti et al. (2005), ao manter-se um forte foco no aspecto interpessoal do processo, pode-se esperar que os processos sociais de influência (Vince, 2001), o sensegiving (Gioia \& Chittipeddi, 1991) e o interpretativismo (Stamatov, 2002) tenham influência importante na quantidade, qualidade e direção da aprendizagem que ocorre no espaço de uma organização. Pode-se esperar também que a aprendizagem esteja intimamente conectada com a identidade em ambos os níveis, individual e organizacional (Cook \& Yanow, 1993).

Mais importante, entretanto, são as novas práticas (formal e informal) e as estruturas que podem ser um dos resultados do processo de aprendizagem (Feldman \& Pentland, 2003). Estes resultados ainda podem ser vistos como manifestações do processo em andamento (em atividade) muito mais do que os resultados finais. Como manifestações e artefatos, contudo, começam influenciando o próprio processo, de uma maneira recursiva. 
A segunda questão é redefinir o link entre $\mathrm{AO}$ e os vários resultados de desempenho em nível de grupo ou organização: muito frequentemente, uma relação positiva é assumida, ou pela definição de aprendizagem ou por sua operacionalização; e isto trivializa o conceito de aprendizagem organizacional.

A terceira questão é separar claramente a noção de aprendizagem da de mudança organizacional, processo ao qual também é freqüentemente associada (Crossan \& Berdrow, 2003; Crossan et al., 1999; Fiol \& Lyles, 1985): enquanto está claro que os conceitos estão relacionados, sua relação é e tem de ser uma questão empírica e dependente de outros fatores, não como algo assumido em nível teórico e universal.

A quarta questão final, possivelmente a mais promissora para o futuro desenvolvimento do conceito, é recuperar o processo peculiar dos temas que cercam a aprendizagem. Frequentemente, a aprendizagem é simplesmente uma caixa-preta, quando a atenção se volta às entradas (inputs), às condições (facilitadores e obstáculos) ou aos resultados (outputs) do processo.

Acredita-se que, recuperando a dimensão de processo de aprendizagem, de forma semelhante ao que Tsoukas e Chia (2002) propõem para mudança organizacional, pode-se obter um desenvolvimento interessante do conceito e dos estudos em AO.

Para a concepção de aprendizagem como processo aberto, entende-se que se possam aplicar algumas das considerações que Tsoukas e Chia (2002) fazem em relação à mudança organizacional. Esses autores sugerem que, para conceber corretamente a mudança como processo, precisa-se trocar de ponto de vista: a mudança de visão como característica permanente da realidade; e a estrutura como epifenômeno da ação humana: um estado passageiro e temporário que imergirá no fluxo da mudança. Assim, mudança é "o retecer das teias das convicções e hábitos de ação dos atores como um resultado de novas experiências obtidas por interações” (p. 570). Consequentemente, mudança é a criação de novas estruturas; porém estas podem não ser permanentes, pelo constante repensar das categorias que ajudam os indivíduos a estruturar sua compreensão de realidade.

Sugere-se, portanto, que para desenvolver e avançar no estabelecimento do conceito e estudos sobre a $\mathrm{AO}$, os pesquisadores precisam lançar seu olhar para o nível interpessoal, para a noção de neutralidade da meta, considerar a mudança sob outra perspectiva e a natureza processual da aprendizagem. Nenhum dos grupos ou perspectivas identificadas na literatura, pode-se afirmar, abarca satisfatoriamente todas estas condições.

Contudo, transferindo-se a noção de processo de mudança sugerida por Tsoukas e Chia (2002) para a realidade organizacional, adquire-se algo próximo à denominada practicing (Dragonetti et al., 2005): um processo emergente de conexão entre atores, sistemas, processos e artefatos. Trata-se de uma das correntes de pensamento no campo da $\mathrm{AO}$ que tem enfatizado a importância das comunidades de prática e a aprendizagem situada, ou seja, a visão de aprendizagem baseada em práticas (Gherardi \& Nicolini, 2001; Lave \& Wenger, 1991). Esta abordagem parece contemplar, pelo menos mais proximamente nesta encruzilhada de perspectivas, as quatro questões anteriormente apontadas neste artigo.

\section{Considerações Finais: a Aprendizagem Baseada em Práticas como Proposta Às QUESTÕES IDENTIFICADAS}

Por meio da análise aqui realizada, de inspiração multiparadigmática, agruparam-se as contribuições prévias identificadas nas perspectivas apresentadas em cinco campos por meio dos quais alguns temas e questões parecem recorrentes. Lançou-se, portanto, alguma luz sobre quatro questões que o conceito de aprendizagem organizacional tem de superar para tornar-ser significativo. 
A proposta da abordagem da aprendizagem baseada em práticas, mencionada no final da seção anterior, focaliza predominantemente a prática como atividade e seu papel nos processos de aprendizagem, tendo sido aperfeiçoada por Nicolini et al. (2003a). Estes pesquisadores propõem a noção de que $\mathrm{AO}$ e conhecimento são fenômenos principalmente sociais e culturais e que estão baseados em prática, a partir de diferentes e influentes perspectivas: sociológica, interpretativocultural, comunidades de prática, teoria da atividade cultural-histórica e actor-network.

Somando-se a esta abordagem, Antonacopoulou (2005) e Dragonetti et al. (2005) propõem uma visão alternativa da aprendizagem (learning-as-practicing), que busca ir além do aprender como prática. Esta perspectiva enfatiza o ensaiar, no sentido de que experimentar e explorar são os aspectos chave da aprendizagem, quando alinhados à reflexão crítica. Os atores refletem sobre suas práticas para entender as conexões entre os aspectos determinantes, as ações e os resultados.

A abordagem da aprendizagem baseada em práticas está preocupada em compreender como o conhecimento é produzido ou transformado por meio de sujeitos com e/ou em atividade e, não necessariamente, como o atingimento de metas pode representar que ocorreu aprendizagem. Há pelo menos três teorias que podem ser consideradas para a construção da noção de aprendizagem baseada em práticas (Antonello, 2008).

A primeira é da participação pela aprendizagem situada (Lave \& Wenger, 1991); a segunda trata da teoria da atividade historicocultural (Engeström, 2001); e a terceira é a teoria ator-rede (Latour, 2005).

Esta perspectiva da aprendizagem não abarca somente ações e atividades, mas também o papel da linguagem e outros artefatos culturais e materiais, a natureza das interações sociais e as respostas tácitas, situadas e quase instintivas de atores nos mundos e redes socialmente construídos nos quais eles residem (Gherardi \& Nicolini, 2000; Nicolini et al., 2003b). Neste ponto, pode-se dizer que a questão anteriormente levantada, do nível de aprendizagem, é contemplada, pois considera os diferentes níveis e as interações dos indivíduos e dos indivíduos com seu ambiente.

Enfatiza também a importância de comunidades de práticos (practitioners), como o espaço onde a dinâmica social de aprendizagem é negociada; além disso, reforça os princípios de interconectividade e interdependência de agência e estrutura, ponto que é central na teoria de estruturação de Giddens (1984) e na teoria de Law e Hassard (1999) denominada ator-rede (Antonello, 2008). Este ponto contempla a questão referente à noção de mudança, ou seja, considera a estrutura como epifenômeno da ação humana.

Esforços para integrar estes fluxos de pesquisa e auxiliar no entendimento mais sistêmico da natureza da prática resultaram em sua nova conceituação. A noção de prática que passa a enfatizar a natureza holística do fenômeno, como fluxo contínuo de múltiplas, contínuas e interconectadas práticas constituem o cerne de sua evolução (Antonacopoulou, 2005).

A coexistência de uma pluralidade de práticas entrelaçadas que coevoluem em interação dinâmica umas com as outras revela que a execução cotidiana da prática se torna um contexto de tensões entre diferentes práticas e os grupos que as incorporam (Antonello, 2008). Porém as tensões capturam forças sociopolíticas e a elasticidade e fluidez do organizar como diferentes processos, que conectam práticas para prover novas possibilidades (Antonacopoulou, 2006).

Conforme Antonello (2008), a teorias baseada em práticas ressalta que a aprendizagem não pode ser considerada somente como processo individual. A aprendizagem é compreendida pelo emergir de relações e interações das pessoas com os elementos sociais e materiais de contextos particulares. Assim, de acordo com a autora, contexto é considerado, a partir de suas divisões do trabalho e relações de poder, ambiente, cultura, linguagem, dentre outros. Aqui revela-se a aprendizagem vista enquanto processo, em todas as suas tensões e fluidez.

Por fim, sugere-se que um possível modo para superar estes desafios. É a adoção de uma perspectiva baseada em práticas (Nicolini et al., 2003b), olhando para os microprocessos que estão 
por trás das práticas contínuas dos atores em um sistema social. Neste contexto, aprender se torna ato reflexivo, por meio de olhar os sistemas por seus atos passados e os planos de seu futuro.

Acredita-se que esta nova conceituação do processo de aprendizagem pode gerar consideráveis insights em sua natureza, contribuindo também ao mesmo tempo para a compreensão da microdinâmica da organização e o fluxo de mudança em um sistema social. Porém, para obter estes insights, é necessário também repensar os métodos que devem fazer parte de nossa caixa de ferramentas, por causa da natureza interpretativa do processo em questão.

Artigo recebido em 06.02.2008. Aprovado em 25.03.2009.

\section{REFERÊNCIAS BIBLIOGRÁFICAS}

Adler, P., \& Clark, K. (1991). Behind the learning curve: a sketch of the learning process. Management Science, 37(3), 267-281.

Alvesson, M., \& Willmott, H. (2002). Identity regulation as organizational control: producing the appropriate individual. Journal of Management Studies, 39(5), 619-644.

Antonacopoulou, E. P. (2005, julho). Strategizing as practising: strategic learning as connecting. EGOS Colloquium, Berlin, Alemanha, 21.

Antonacopoulou, E. P. (2006). The relationship between individual and organizational learning: new evidence from managerial learning practices. Management Learning, 37(4), 455-473.

Antonacopoulou, E. P., \& Gabriel, Y. (2001). Emotion, learning and organisational change: towards and integration of psychoanalytic end other perspectives. Journal of Organisational Change Management, 14(5), 435-451.

Antonello, C. S. (2002, setembro). Estudo dos métodos e posicionamento epistemológico na pesquisa de aprendizagem organizacional, competências e gestão do conhecimento. Encontro Nacional da Associação Nacional de Pós-Graduação e Pesquisa em Administração, Salvador, BA, Brasil, 26.

Antonello, C. S. (2005). A metamorfose da aprendizagem organizacional: uma revisão crítica. In R. Ruas, C. S. Antonello, \& L. H. Boff (Orgs.), Aprendizagem organizacional e competências (pp. 12-33). Porto Alegre: Artmed.

Antonello, C. S. (2008). Aprendizagem nas organizações: refletindo sobre suas abordagens [Mimeo]. Universidade Federal do Rio Grande do Sul, Porto Alegre, RS.

Argote, L. (1999). Organizational learning: creating, retaining and transferring knowledge. Boston: Kluwer Academic Publishers.

Argyris, C. (1990). Overcoming organizational defences. Boston: Allyn and Bacon.

Argyris, C., \& Schön, D. (1978). Organizational learning: a theory of action perspective. Reading, Massachusetts: Addison-Wesley.

Bapuji, H., \& Crossan, M. (2004). From questions to answers: reviewing organizational learning research. Management Learning, 35(4), 397-417.

Bell, S., Whitwell, G., \& Lukas, B. (2002). Schools of thought in organizational learning. Journal of the Academy of Marketing Science, 30(1), 70-86. 
Berthoin Antal, A., Dierkes, M., Child, J., \& Nonaka, I. (2001). Organizational learning and knowledge: reflections on the dynamics of the field and challenges for the future. In M. Dierkes, A. Berthoin Antal, J. Child, \& I. Nonaka (Orgs.), The handbook of organizational learning and knowledge (pp. 921-939). Oxford: Oxford University Press.

Boerner, C., Macher, J., \& Teece, D. (2001). A review and assessment of organizational learning in economic theories. In M. Dierkes, A. Berthoin Antal, J. Child, \& I. Nonaka (Orgs.), The handbook of organizational learning and knowledge (pp. 89-117). Oxford: Oxford University Press.

Bontis, N., Crossan, M., \& Hulland, J. (2002). Managing an organizational learning system by aligning stocks and flows. Journal of Management Studies, 39(4), 438-469.

Brown, J., \& Duguid, P. (1991). Organizational learning and communities-of-practice: towards a unified view of working, learning and innovation. Organization Science, 2(1), 40-57.

Bryman, A. (2004). Social research methods (2a ed.). Oxford: University Press.

Cangelosi, V., \& Dill, W. (1965). Organizational learning: observations towards a theory. Administrative Science Quarterly, 10(2), 175-203.

Collins, R. (1994). Four sociological traditions. New York: Oxford University Press.

Contu, A., Grey, C., \& Örtenblad, A. (2003). Against learning. Human Relations, 56(8), 931-952.

Cook, S. D. N., \& Yanow, D. (1993). Culture and organizational learning. Journal of Management Inquiry, 2(4), 373-390.

Corley K., \& Gioia, D. (2003) Semantic learning as change enabler: relating organizational identity and organizational learning. In M. Easterby-Smith \& M. A. Lyles (Orgs.), Handbook of organizational learning and knowledge management (pp. 623-638). Oxford: Blackwell.

Crossan, M., \& Berdrow, I. (2003). Organizational learning and strategic renewal. Strategic Management Journal, 24(11), 1087-1105.

Crossan, M., \& Guatto, T. (1996). Organizational learning research profile. Journal of Organizational Change Management, 9(1), 107-12.

Crossan, M., Lane, H., \& White, R. (1999). An organizational learning framework: from intuition to institution. Academy of Management Review, 24(3), 522-537.

Cyert, R., \& March, J. (1992). A behavioral theory of the firm (2nd ed.). Cambridge: Blackwell.

Czarniawska, B. (2001). Anthropology and organizational learning. In M. Dierkes, A. Berthoin Antal, J. Child, \& I. Nonaka (Orgs.), The handbook of organizational learning and knowledge (pp. 118-136). Oxford: Oxford University Press.

Daft, R., \& Weick, K. (1984). Toward a model of organizations as interpretation systems. Academy of Management Review, 9(2), 284-295.

Dewey, J. (1938). Experience \& education. New York: Macmillan.

Dierkes, M., Berthoin Anthal, A., Child, J., \& Nonaka, I. (2001). Handbook of organizational learning and knowledge. Oxford: Oxford University Press.

Dodgson, M. (1993). Organizational learning - a review of some literatures. Organization Studies, 14(3), 375-94. 
Dosi, G., \& Orsenigo, L. (1988). Coordenation and transformation: an overview on structures, behaviours and change in evolutionary enviroments. In G. Dosi, C. Freeman, R. Nelson, G. Silverman, \& L. Soete (Orgs.), Technical change and economic theory (pp. 13-37). London: Printer.

Dragonetti, N. C., Antonacopoulou, E. P., Broekhuizen, M. C. S., \& Patnaik, S. (2005). Organisational learning: a review, a critique and a way forward. Proceedings of the Organization studies workshop theorizing process in organizational research, Santorini, Itália, 1.

Easterby-Smith, M. (1997). Disciplines of organizational learning: contributions and critiques. Human Relations, 50(9), 1085-113.

Easterby-Smith, M., \& Lyles, M. (2003) The blackwell handbook of organizational learning and knowledge management. Oxford: Blackwell Publishing.

Edmondson, A., \& Moingeon, B. (1998). From organizational learning to the learning organization. Management Learning, 29(1), 5-20.

Engeström, Y. (2001). Expansive learning at work: toward an activity theoretical reconceptualization. Journal of Education and Work, 14(1), 133-156.

Fear, J. R. (2001). Thinking historically about organizational learning. In M. Dierkes, A. Berthoin Antal, J. Child, \& I. Nonaka (Orgs.), The handbook of organizational learning and knowledge (pp.162-191). Oxford: Oxford University Press.

Feldman, M., \& Pentland, B. (2003). Reconceptualizing organizational routines as a source of flexibility and change. Administrative Science Quarterly, 48(1), 94-118.

Fiol, C. M., \& Lyles, M. (1985). Organizational learning. Academy of Management Review, 10(4), 803-813.

Forrester, J. W. (1975). Learning through system dynamics as preparation for de 21st century. In J. W. Forrester. Collected papers of J. W. Forrester (pp. 211-244). Portland: Productivity Press.

Garvin, D. (1993). Building a learning organization. Harvard Business Review, 71(4), 78-91.

Gavetti, G., \& Levinthal, D. (2000). Looking forward and looking backward: cognitive and experiential search. Administrative Science Quarterly, 45(1), 113-137.

Gephart, R. (1999). Paradigms and research methods [Research Methods Forum]. Recuperado em 1 agosto, 2004, de http://division.aomonline.org/rm/1999_RMD_Forum_Paradigms_and_Research_Methods.htm

Gherardi, S., \& Nicolini, D. (2000). The organizational learning of safety in communities of practice. Journal of Management Inquiry, 9(1), 7-18.

Gherardi, S., \& Nicolini, D. (2001). The sociological foundations of organizational learning. In M. Dierkes, A. Berthoin Antal, J. Child, \& I. Nonaka (Orgs.), The handbook of organizational learning and knowledge (pp.35-60). Oxford: Oxford University Press.

Giddens, A. (1984). The constitution of society. Cambridge: Polity Press.

Gioia, D., \& Chittipeddi, K. (1991). Sensemaking and sensegiving in strategic change initiation. Strategic Management Journal, 12(6), 433-448.

Gioia, D. A., \& Pitre, E. (1990). Multiparadigm perspectives on theory building. Academy of Management Review, 15(4), 584-602.

Hedberg, B. (1981). How organisations learn and unlearn. In P. Nyström \& W. Starbuck (Orgs.), Handbook of organizational design (pp. 8-27). New York: Oxford University Press. 
Huber, G. P. (1991). Organizational learning: the contributing processes and the literatures. Organization Science, 2(1), 88-115.

Kolb, D. A. (1984). Experimental learning: experience as the source of learning. New Jersey: Prentice-Hall.

Krogh, V. G. von, Roos, J., \& Slocum, K. (1996). An essay on corporate epistemology. In G. von Krogh \& J. Roos (Orgs.), Managing knowledge (pp. 157-183). London: Sage.

Lant, T. K. (2002). Organizational cognition and interpretation. In J. Baum (Org.), The blackwell companion to organization (pp. 344-362). Oxford: Blackwell.

Lapalombara, J. (2001). The underestimated contributions of political science to organizational learning. In M. Dierkes, A. Berthoin Antal, J. Child, \& I. Nonaka (Orgs.), The handbook of organizational learning and knowledge (pp. 137-161). Oxford: Oxford University Press.

Latour, B. (2005). Re-assembling the social - an introduction to actor network theory. London: Oxford University Press.

Lave, J., \& Wenger, E. (1991). Situated learning: legitimate peripheral participation. New York: Cambridge University Press.

Law, J., \& Hassard, J. (1999). Actor network theory and after. Oxford, UK: Blackwell.

Lawrence, T., Mauws, M. K., Dyck, B., \& Kleysen, R. F. (2005). The politics of organizational learning: integrating power into the 4I framework. Academy of Management Review, 30(1), 180-191.

Levitt, B., \& March, J. (1988). Organizational learning. Annual Review of Sociology, 14, 319-340.

Lewin, K. (1947). Frontiers in group dynamics: II. Channel of group life: social planning and action research. Human Relations, 1(2), 143-153.

Lewis, M. W., \& Grimes, A. J. (2005). Metatriangulação: a construção de teorias a partir de múltiplos paradigmas. Revista de Administração de Empresas, 45(1), 72-91.

Loiola, E., \& Bastos, A. V. B. (2003). A produção acadêmica sobre aprendizagem organizacional no Brasil. Revista de Administração Contemporânea, 7(3), 181-201.

Lyles, M., \& Easterby-Smith, M. (2003). Organizational learning and knowledge management: agendas for future research. In M. Easterby-Smith \& M. A. Lyles (Orgs.), Handbook of organizational learning and knowledge management (pp. 639-652). Oxford: Blackwell.

Maier, G., Prange, C., \& Rosenstiel, L. von. (2001). Psychological perspectives of organizational learning. In M. Dierkes, A. Berthoin Antal, J. Child, \& I. Nonaka (Orgs.), The handbook of organizational learning and knowledge (pp. 14-34). Oxford: Oxford University Press.

March, J., \& Simon, H. (1993). Organizations (2nd ed.). Cambridge: Blackwell.

Mezirow, W. (1991). Transformative dimensions of adult learning. San Francisco: Jossey-Bass.

Miller, D. (1996). A preliminary typology of organizational learning: synthesizing the literature. Journal of Management, 22(3), 485-505.

Nelson, R., \& Winter, S. (1982). An evolutionary theory of economic change. Cambridge: Belknap.

Nicolini, D., Gherardi, S., \& Yanow, D. (2003a). Introduction: towards a practice-based view of knowing and learning in organizations. In D. Nicolini, S. Gherardi, \& D. Yanow (Orgs.), Knowing in organizations: a practice-based approach (pp. 3-31). London: Sharpe. 
Nicolini, D., Gherardi, S., \& Yanow, D. (2003b). Knowing in organizations: a practice-based approach. London: Sharpe.

Nonaka, I., \& Takeuchi, H. (1997). Criação do conhecimento na empresa - como as empresas japonesas geram a dinâmica da inovação. Rio de Janeiro: Campus.

Orr, J. (1990). Talking about machines: an ethnography of a modern job. Ph.D. Thesis, Cornell University, Ithaca, NY, USA.

Pavitt, K. (1987). The objectives of technology policy. Science and Public Policy, 14(4), 182-188.

Pawlowsky, P. (2001). Management science and organizational learning. In M. Dierkes, A. Berthoin Antal, J. Child, \& I. Nonaka (Orgs.), The handbook of organizational learning and knowledge (pp. 61-88). Oxford: Oxford University Press.

Pedler, M., Burgoyne, J., \& Boydell, T. (1991). The learning company. New York: McGraw-Hill.

Piaget, J. (1978). A epistemologia genética; sabedoria e ilusões da filosofia; problemas de psicologia genética. São Paulo: Abril Cultural.

Raelin, J. A. (2001). Public reflection as the basis of learning. Management Learning, 32(1), 11-30.

Revans, R. W. (1980). Action learning—new techniques for management. London: Blond and Briggs.

Revans, R. W. (1982). The enterprise as a learning system. In R. W. Revans (Org.), The origins and growth of action learning. Goch: Chartwell Bratt.

Rodrigues, S., Child, J., \& Luz, T. (2003). Aprendizagem contestada. Revista de Administração de Empresas, 44(1), 27-43.

Schein, E. H. (1991). What is culture? In P. J. Frost, L. F. Moore, M. R. Louis, C. C. Lundberg, J. Martin (Orgs.), Reframing Organizational Culture (pp. 243-53). Newbury Park: Sage.

Schön, D. A. (1983). The reflective practitioner. New York: Arena.

Schultz, M., \& Hatch, M. (1996). Living with multiple paradigms: the case of paradigm interplay in organizational culture studies. Academy of Management Review, 21(2), 529-557.

Senge, P. M. (1990). A quinta disciplina. São Paulo: Best Seller.

Shrivastava, P. (1983). A typology of organizational learning systems. Journal of Managenent Studies, 20(1), 7-28.

Slater, S. F., \& Narver, J. C. (1995). Market orientation and the learning organization. Journal of Marketing, 59(3), 63-74.

Stamatov, P. (2002). Interpretive activism and the political uses of Verdi's operas in the 1840s. American Sociological Review, 67(3), 345-366.

Tenkasi, R., \& Boland, R. (1996). Exploring knowledge diversity in knowledge intensive firms. Journal of Organizational Change Management, 9(1), 79-91.

Tsang, E. W. K. (1997). Organizational learning and the learning organization: a dichotomy between descriptive and prescriptive research. Human Relations, 50(1), 73-89.

Tsoukas, H., \& Chia, R. (2002). On organizational becoming: rethinking organizational change. Organization Science, 13(5), 567-582.

Ulrich, H., \& Probst, G. J. B. (1990). Anleitung zum ganzheitlichen denken und handeln. Bern: Haupt. 
Vera, D., \& Crossan, M. (2003). Organizational learning and knowledge management: toward an integrative framework. In M. Easterby-Smith \& M. A. Lyles (Orgs.), Handbook of organizational learning and knowledge management (pp.122-141) Oxford: Blackwell Publishing.

Vince, R. (2001). Power and emotion in organizational learning. Human Relations, 54(10), 13251351.

Walsh, J., \& Ungson, G. R. (1991). Organizational memory. Academy of Management Review, 16(1), 57-91.

Weber, C., \& Berthoin Antal, A. (2001). The role of time in organizational learning. In M. Dierkes, A. Berthoin Antal, J. Child, \& I. Nonaka (Orgs.), The handbook of organizational learning and knowledge (pp. 351-368). Oxford: Oxford University Press.

Weick, K., \& Westley, F. (1996). Organizational learning: affirming an oxymoron. In S. Clegg, C. Hardy, \& W. Nord (Orgs.), Handbook of organization studies (pp. 440-458). London: Sage.

Wiegand, M. (1996). Prozesse organisationalen lernens. Wiesbaden: Gabler.

Winter, S. G. (2000). The satisficing principle in capability learning. Strategic Management Journal, 21(10-11), 981-996. 